\title{
Effect of Coronary Computed Tomography Angiography Disease Burden on the Incidence of Recurrent Chest Pain
}

\author{
Homayoun R. Ahmadian, Dustin M. Thomas, David J. Shaw, Megan L. Barnwell, \\ Ronald L. Jones, Ryan J. McDonough, Ryan L. Prentice, Charles K. Lin, and Ahmad M. Slim
}

Cardiology Service MCHE-MDC, Brooke Army Medical Center, 3551 Roger Brooke Drive, San Antonio, TX 78234-6200, USA

Correspondence should be addressed to Ahmad M. Slim; ahmad.m.slim.mil@mail.mil

Received 3 March 2014; Revised 17 April 2014; Accepted 18 April 2014; Published 2 July 2014

Academic Editor: Chien-Feng Li

Copyright (C) 2014 Homayoun R. Ahmadian et al. This is an open access article distributed under the Creative Commons Attribution License, which permits unrestricted use, distribution, and reproduction in any medium, provided the original work is properly cited.

\begin{abstract}
Introduction. The purpose of this study is to investigate chest pain evaluations after initial coronary computed tomography angiography (CCTA) based upon coronary artery disease (CAD) burden. Methods. CCTA results of 1,518 patients were grouped based on the CCTA results into no CAD, nonobstructive CAD ( $<50 \%$ maximal diameter stenosis), or obstructive CAD ( $\geq 50 \%$ stenosis). Chest pain evaluation after initial CCTA and rates of major adverse cardiovascular events (MACE) defined as the incidence of all-cause mortality, nonfatal MI, ischemic stroke, and late revascularization ( $>90$ days following CCTA) were evaluated. Results. MACE rates were higher with obstructive CAD compared to nonobstructive CAD and no CAD $(8.9 \%$ versus $0.7 \%$, $P<0.001 ; 8.9$ versus $1.6 \%, P<0.001)$. One hundred seventy-four patients $(11.5 \%)$ underwent evaluation for chest pain after index CCTA with rates significantly higher with obstructive CAD compared to both nonobstructive CAD and no CAD (7.5\% versus $13.9 \%$ versus $17.8 \%, P<0.001$ ). The incidence of repeat testing was more frequent in patients with obstructive CAD (no CAD $36.5 \%$ versus nonobstructive CAD 54.9\% versus obstructive CAD 67.7\%, $P=0.015)$. Conclusion. Absence of obstructive disease on CCTA is associated with lower rates of subsequent evaluations for chest pain and repeat testing with low MACE event rates over a 22-month followup.
\end{abstract}

\section{Introduction}

Coronary CT angiography (CCTA) has emerged as an effective tool for both outpatient and emergency department (ED) evaluation of low to intermediate risk patients presenting with acute chest pain syndromes [1-6]. CCTA has also been shown effective as a complementary test in patients with equivocal or abnormal noninvasive results without high risk features [7]. The high negative predictive value ( 99\%) associated with maximal lumen diameter stenosis values $<50 \%$ allows for safe disposition and potentially avoids the cost and risk associated with invasive coronary angiography (ICA) [8]. This has resulted in guideline statements recommending CCTA for these indications [9]. Recent updates to hospital inpatient reimbursement emphasize minimizing short stay inpatient hospitalizations under which chest pain rule-outs frequently fall [10]. This places a premium on the safe and efficient evaluation of this common symptom presentation that is cost effective.
Recurrent chest pain presentations often lead to repeat testing and recurrent hospitalizations. Recurrent emergency department evaluations and hospitalizations for chest pain have been reported previously without commenting on CCTA disease burden [11, 12]. We attempt to investigate recurrent chest pain evaluation after initial evaluation with coronary computed tomography angiography (CCTA) between those with previous obstructive CAD versus those with nonobstructive or no CAD as well as a comparison for major cardiac adverse events (MACE) in these groups.

\section{Methods}

Coronary CT angiography (CCTA) results from January 2005 until July 2012 at a high volume, single center tertiary referral hospital were retrospectively reviewed. A study population of 1518 patients evaluated in emergency department (ED), inpatient, or outpatient setting for the indications of chest 
TABLE 1: Baseline demographic data and chest reevaluation outcomes based on CCTA disease burden.

\begin{tabular}{|c|c|c|c|c|}
\hline & $\begin{array}{c}\text { No CAD } \\
n=689(\%)\end{array}$ & $\begin{array}{c}\text { Nonobstructive CAD } \\
n=655(\%)\end{array}$ & $\begin{array}{c}\text { Obstructive CAD } \\
n=174(\%)\end{array}$ & $P$ value \\
\hline Age, years & $51.8 \pm 13.9$ & $60.9 \pm 12.6$ & $72.9 \pm 17.7$ & $<0.001$ \\
\hline Male & $410(59.5)$ & $391(59.7)$ & $118(67.8)$ & 0.113 \\
\hline Reevaluation for chest pain & $52(7.5)$ & $91(13.9)$ & $31(17.8)$ & $<0.001$ \\
\hline Diabetes mellitus & $14(2.0 \%)$ & $31(4.7 \%)$ & $17(9.8 \%)$ & $<0.001$ \\
\hline Hypertension & $155(22.5 \%)$ & $307(46.9 \%)$ & $98(56.3 \%)$ & $<0.001$ \\
\hline Hyperlipidemia & $129(18.7 \%)$ & $266(40.6)$ & $72(41.4 \%)$ & $<0.001$ \\
\hline All-cause mortality & $2(0.3 \%)$ & $0(0.0 \%)$ & $1(0.6 \%)$ & 1.000 \\
\hline Stroke & $3(0.4 \%)$ & $4(0.6 \%)$ & $6(3.4 \%)$ & $<0.001$ \\
\hline Myocardial infarction & $0(0.0 \%)$ & $6(0.9 \%)$ & $4(2.3 \%)$ & 0.003 \\
\hline Late revascularization & $0(0.0 \%)$ & $1(0.2 \%)$ & $4(2.3 \%)$ & $<0.001$ \\
\hline Composite MACE & $5(0.7 \%)$ & $11(1.7 \%)$ & $14(8.0 \%)$ & $<0.001$ \\
\hline
\end{tabular}

pain, dyspnea, syncope, evaluation of graft/stent patency, or possible anomalous coronary artery was identified. Patients were grouped based on the CCTA results demonstrating either no CAD, nonobstructive CAD ( $<50 \%$ maximal diameter stenosis), or obstructive CAD ( $\geq 50 \%$ stenosis). Recurrent chest pain was determined based on ICD-9 codes. Rates of major adverse cardiovascular events (MACE) were abstracted.

CCTA images were analyzed by a cardiologist with level III ACC/ACR certified imaging expert in accordance with SCCT guidelines [13]. From January 2005 to December 2007, images were obtained using a 16-slice CT scanner (Brilliance16R, Phillips, Amsterdam, Netherlands). From January 2008 to March 2011, images were obtained using a retrospective helical protocol with a 64 slice CT scanner (Somatom Definition CTR, Siemens, Erlagen, Germany). From March 2011 to March 2012, images were obtained utilizing a prospective sequential protocol with $60-80 \%$ image acquisition window. In March 2012 to July 2012, images were obtained using a 128-slice dual head scanner with a single heart beat image acquisition of the complete coronary when a heart rate of less than 60 was achieved (Somatom Definition Flash CTR, Siemens, Erlagen, Germany).

The primary endpoint was recurrent chest pain evaluation. Recurrent chest pain evaluations were identified initially by searching ICD-9 codes for atypical chest pain (786.59), chest pain (786.5), and angina (413) both in the inpatient and outpatient setting. All identified chest pain diagnoses after initial CCTA were manually adjudicated by per patient chart review. Any additional invasive or noninvasive cardiac risk stratification testing performed after the initial CCTA was documented, including multiple testing when applicable. Centers for Medicare and Medicaid Services (CMS) reimbursement rates, released in December 2013, were used to calculate per patient cost for additional risk stratification testing following CCTA. The composite secondary outcome was MACE, defined as all-cause mortality, stroke, nonfatal $\mathrm{MI}$, and late revascularization, defined as revascularization performed within 90 days of CCTA with CAC imaging. ICD-9 codes for all-cause mortality $(798.1,798.2,798.9$, and
V12.53), stroke (434.00, 434.01, 434.10, 434.11, 434.90, 434.91, 997.02, and V12.54), nonfatal MI (410.0-410.9), and late revascularization with PCI (92980, 92981, 92982, 92995, and 92996) or CABG (33510-33514, 33516, and 33533-33536) were used for initial data extraction followed by Department of Defense (DOD) outpatient and inpatient electronic medical records (EMR) verification of events. We determined mortality using the social security death index (SSDI) followed by reverification using EMR for last visit date as well as Tricare Healthcare Informatics Division verification. All events identified by ICD-9 code were adjudicated.

Statistical analysis was performed using IBM SPSS version 19.0 (IBM, Arnock, New York). Continuous variables are presented as means \pm standard deviation. Categorical variables are presented as frequencies with percentages. Comparison of means between the various CCTA groups was performed using one-way ANOVA with $P$ values $<0.05$ considered statistically significant. Kaplan-Meier method was used to demonstrate chest pain-free survival in the recurrent chest pain population as well as total survival free from MACE in the total population with a log rank value $<0.05$ considered statistically significant.

\section{Results}

One thousand five hundred eighteen patients underwent CCTA in an ED, inpatient or outpatient setting. Table 1 shows the baseline demographic data and outcomes for the total patient cohort. Gender was equally distributed amongst the groups; however, the mean age increased with increasing CCTA disease burden $(P<0.001)$. CCTA evaluation in these 1518 patients at index evaluation demonstrated obstructive CAD, nonobstructive CAD, and no CAD in $11.5 \%, 43.1 \%$, and $45.4 \%$, respectively. Patients with obstructive CAD on CCTA were more likely to have documented diabetes mellitus $(P<0.001)$ and hypertension $(P<0.001)$. Obstructive CAD and nonobstructive CAD patients were more likely to have hyperlipidemia than no CAD CCTA patients $(P<0.001)$. 
TABLE 2: Reevaluations for chest pain based on CCTA disease burden.

\begin{tabular}{|c|c|c|c|c|}
\hline & $\begin{array}{c}\text { No CAD } \\
n=52\end{array}$ & $\begin{array}{c}\text { Nonobstructive } \\
\text { CAD } \\
n=91\end{array}$ & $\begin{array}{c}\text { Obstructive CAD } \\
n=31\end{array}$ & $P$ value \\
\hline Age, years & $52.8 \pm 11.7$ & $58.8 \pm 13.5$ & $56.4 \pm 12.7$ & 0.029 \\
\hline Male, $n(\%)$ & $23(44.2)$ & $46(50.5)$ & $18(58.1)$ & 0.470 \\
\hline Repeat testing, $n(\%)$ & $19(36.5)$ & $50(54.9)$ & $21(67.7)$ & 0.015 \\
\hline Repeat tests per patient & $0.4 \pm 0.5$ & $0.7 \pm 0.7$ & $0.8 \pm 0.7$ & 0.006 \\
\hline Cost of additional testing per patient & $\$ 98$ & $\$ 226$ & $\$ 255$ & 0.001 \\
\hline Mean time to 1st reevaluation, days & $348 \pm 375$ & $355 \pm 314$ & $330 \pm 332$ & 0.938 \\
\hline Hospitalization at 1st reevaluation, $n(\%)$ & $18(34.6)$ & $49(53.8)$ & $18(58.1)$ & 0.046 \\
\hline 1st reevaluation mean LOS, days & $1.2 \pm 0.5$ & $1.3 \pm 0.7$ & $1.6 \pm 1.0$ & 0.810 \\
\hline 2nd reevaluation, $n(\%)$ & $19(36.5)$ & $30(33.0)$ & $8(25.8)$ & 0.601 \\
\hline Mean time to 2 nd reevaluation, days & $567 \pm 410$ & $581 \pm 301$ & $439 \pm 322$ & 0.580 \\
\hline Hospitalizations at 2 nd reevaluation, $n$ & $8(15.4)$ & $11(12.1)$ & $5(16.1)$ & 0.788 \\
\hline 2nd reevaluation mean LOS, days & $1.4 \pm 0.7$ & $1.6 \pm 1.0$ & $1.8 \pm 1.8$ & 0.810 \\
\hline 3rd reevaluation, $n(\%)$ & $4(7.7)$ & $8(8.8)$ & $3(9.7)$ & 0.949 \\
\hline Mean time to 3rd reevaluation, days & $592 \pm 451$ & $658 \pm 425$ & $485 \pm 461$ & 0.843 \\
\hline Hospitalizations at 3 rd reevaluation, $n$ & $0(0)$ & $0(0)$ & $0(0)$ & $\mathrm{n} / \mathrm{a}$ \\
\hline 3rd reevaluation mean LOS, days & $\mathrm{n} / \mathrm{a}$ & $1.0 \pm 0.0$ & $1.0 \pm 0.0$ & 1.000 \\
\hline 4 th reevaluation, $n$ & $0(0)$ & $3(3.3)$ & $1(3.2)$ & 0.418 \\
\hline Mean time to 4 th reevaluation, days & $\mathrm{n} / \mathrm{a}$ & $1008 \pm 354$ & $180 \pm 0$ & 0.180 \\
\hline Hospitalizations at 4 th reevaluation, $n$ & 0 & $2(3.3)$ & 0 & 0.249 \\
\hline 4th reevaluation mean LOS, days & $\mathrm{n} / \mathrm{a}$ & $1.0 \pm 0.0$ & $\mathrm{n} / \mathrm{a}$ & $\mathrm{n} / \mathrm{a}$ \\
\hline
\end{tabular}

Of the 174 patients with obstructive disease, single-vessel, 2vessel, and 3-vessel obstruction was found in 64.5\%, 32.3\%, and $3.2 \%$, respectively. All-cause mortality was rare in this patient population with 3 total deaths and was not statistically different stratified by CAD burden. Stroke, nonfatal MI, late revascularization, and composite MACE rates were significantly higher in the obstructive CAD cohort $(P$ values $<0.001$, $0.003,<0.001$, and $<0.001$, resp.) with overall low event rates.

Recurrent chest pain evaluation occurred in 174 patients (11.5\%) overall. Fifty-two patients with no CAD, 91 patients with nonobstructive $\mathrm{CAD}$, and 31 patients with obstructive CAD (Table 2) presented for recurrent chest pain evaluation ( $7.5 \%$ versus $13.9 \%$ versus $17.8 \%, P<0.001)$. The mean age at the time of reevaluation differed amongst the groups; however the nonobstructive CAD patients tended to be older at time of reevaluation (mean age 58.8 years, $P=0.029$ ). The incidence of repeat testing (Table 2 ) was more frequent in patients with obstructive CAD (no CAD $36.5 \%$ versus nonobstructive CAD 54.9\% versus obstructive CAD $67.7 \%, P=0.015)$. This correlated with increased frequency of hospitalizations at initial reevaluation amongst the groups with $34.6 \%, 53.8 \%$, and $58.1 \%$ of patients in the no $\mathrm{CAD}$, nonobstructive $\mathrm{CAD}$, and obstructive CAD groups, respectively $(P=0.046)$. There was no difference observed with respect to the mean duration of time between index CCTA and reevaluation for chest pain $(P=0.938)$ nor the length of stay (LOS) during hospitalization at first reevaluation $(P=0.810)$. At the time of reevaluation, review of initial CCTA results was significantly correlated with time free of recurrent chest pain with those having no CAD having a longer chest pain-free survival period with $\log$ rank showing $P<0.001$ (Figure 1). Health care reevaluations for chest pain, including second, third, and fourth additional evaluations, were evaluated and were found not to be statistically significant amongst the three CCTA disease groups. Overall MACE free survival in the chest pain reevaluation cohort was significant for more events in the obstructive CAD group when compared to nonobstructive $\mathrm{CAD}$ and no CAD (log rank = 0.021) (Figure 2).

Outcomes and ability to predict recurrent chest pain evaluations and MACE were broken down into individual major epicardial coronary vessels and analyzed with respect to CAD burden. Obstructive LM CAD (Table 3) on initial CCTA was associated with higher rates of hospitalization at reevaluation $(P=0.032)$, high per patient cost in repeat testing $(P=0.019)$, higher all-cause mortality $(P<0.001)$, and composite MACE rates $(P<0.001)$. There was no difference between nonobstructive LM CAD, obstructive LM CAD, and no LM CAD with respect to chest pain reevaluations $(13.5 \%$ versus $14.3 \%$ versus $11.1 \%$, resp. $P=$ 0.563). Nonobstructive LM CAD was associated with longer hospital LOS (1.8 \pm 1.3 days) when compared with no LM CAD and obstructive LM CAD $(1.2 \pm 0.5$ days versus $1.0 \pm$ 0.0 days, $P=0.005$ ).

Obstructive LAD CAD (Table 4) was associated with higher rates of chest pain reevaluation $(P<0.001)$, hospitalization at reevaluation $(P<0.001)$, repeat testing at the time of reevaluation $(P<0.001)$, average per patient 
TABLE 3: Baseline demographic data, outcomes, and cost analysis based on burden of CAD in the LM.

\begin{tabular}{|c|c|c|c|c|}
\hline & $\begin{array}{c}\text { No LM CAD } \\
n=1289\end{array}$ & $\begin{array}{c}\text { Nonobstructive LM } \\
n=222\end{array}$ & $\begin{array}{c}\text { Obstructive LM } \\
\quad n=7\end{array}$ & $P$ value \\
\hline Age & $51.8 \pm 13.9$ & $60.9 \pm 12.6$ & $72.9 \pm 17.7$ & $<0.001$ \\
\hline Male gender & $774(60)$ & $139(62.6)$ & $6(85.7)$ & 0.303 \\
\hline Chest pain reevaluation, $n(\%)$ & $143(11.1)$ & $30(13.5)$ & $1(14.3)$ & 0.563 \\
\hline Time to reevaluation, days & $353 \pm 342$ & $335 \pm 304$ & $100 \pm 0$ & 0.732 \\
\hline Hospitalization at reevaluation, $n(\%)$ & $64(5.0)$ & $20(9.0)$ & $1(14.3)$ & 0.032 \\
\hline Hospital LOS, days & $1.2 \pm 0.5$ & $1.8 \pm 1.3$ & $1.0 \pm 0$ & 0.005 \\
\hline Repeat testing, $n(\%)$ & $70(5.4)$ & $19(8.6)$ & $1(14.3)$ & 0.122 \\
\hline Tests per patient, mean & $0.06 \pm 0.3$ & $0.1 \pm 0.4$ & $0.1 \pm 0.4$ & 0.131 \\
\hline Cost per patient, mean & $\$ 18 \pm 100$ & $\$ 37 \pm 139$ & $\$ 83 \pm 220$ & 0.019 \\
\hline All-cause mortality, $n(\%)$ & $3(0.2)$ & $0(0)$ & $1(14.3)$ & $<0.001$ \\
\hline Stroke, $n(\%)$ & $8(0.6)$ & $5(2.3)$ & $0(0)$ & 0.05 \\
\hline $\mathrm{MI}, n(\%)$ & $9(0.7)$ & $7(3.2)$ & $0(0)$ & 0.004 \\
\hline Late revascularization, $n(\%)$ & $2(0.2)$ & $3(1.4)$ & $0(0)$ & 0.016 \\
\hline Composite MACE, $n(\%)$ & $20(1.6)$ & $13(5.9)$ & $1(14.3)$ & $<0.001$ \\
\hline
\end{tabular}

LM: left main coronary artery.

TABLE 4: Baseline demographic data, outcomes, and cost analysis based on burden of CAD in the LAD.

\begin{tabular}{|c|c|c|c|c|}
\hline & $\begin{array}{c}\text { No LAD CAD } \\
n=747\end{array}$ & $\begin{array}{c}\text { Nonobstructive LAD } \\
n=631\end{array}$ & $\begin{array}{c}\text { Obstructive LAD } \\
n=140\end{array}$ & $P$ value \\
\hline Age & $48.6 \pm 14.1$ & $57.4 \pm 12.9$ & $58.6 \pm 12.2$ & $<0.001$ \\
\hline Male gender & $448(60)$ & $380(60.2)$ & $91(65)$ & 0.524 \\
\hline Chest pain reevaluation, $n(\%)$ & $63(8.4)$ & $85(13.5)$ & $26(18.6)$ & $<0.001$ \\
\hline Time to reevaluation, days & $358 \pm 368$ & $353 \pm 308$ & $309 \pm 347$ & 0.804 \\
\hline Hospitalization at reevaluation, $n(\%)$ & $23(3.1)$ & $46(7.3)$ & $16(11.4)$ & $<0.001$ \\
\hline Hospital LOS, days & $1.2 \pm 0.5$ & $1.3 \pm 0.8$ & $1.5 \pm 1.1$ & 0.436 \\
\hline Repeat testing, $n(\%)$ & $23(3.1)$ & $49(7.8)$ & $18(12.9)$ & $<0.001$ \\
\hline Tests per patient, mean & $0.03 \pm 0.2$ & $0.09 \pm 0.3$ & $0.16 \pm 0.5$ & $<0.001$ \\
\hline Cost per patient, mean & $\$ 9 \pm 65$ & $\$ 34 \pm 134$ & $\$ 50 \pm 168$ & $<0.001$ \\
\hline All-cause mortality, $n(\%)$ & $3(0.4)$ & $0(0)$ & $1(0.7)$ & 0.193 \\
\hline Stroke, $n(\%)$ & $3(0.4)$ & $7(1.1)$ & $3(2.1)$ & 0.081 \\
\hline MI, $n(\%)$ & $3(0.4)$ & $11(1.7)$ & $2(1.4)$ & 0.047 \\
\hline Late revascularization, $n(\%)$ & $0(0)$ & $3(0.5)$ & $2(1.4)$ & 0.006 \\
\hline Composite MACE, $n(\%)$ & $9(1.2)$ & $17(2.7)$ & $8(5.7)$ & 0.005 \\
\hline
\end{tabular}

LAD: left anterior descending.

testing and cost $(P<0.001$ for both $)$, rates of myocardial infarction $(P=0.006)$, late revascularization $(P=0.006)$, and composite MACE $(P=0.005)$.

Obstructive LCX CAD (Table 5) was found to be associated with higher rates of chest pain reevaluation $(P=$ 0.048), hospitalization at reevaluation $(P=0.039)$, repeat testing at the time of reevaluation $(P=0.003)$, average per patient testing $(P=0.001)$, average per patient cost for repeat testing $(P<0.001)$, all-cause mortality $(P=0.027)$, stroke $(P=0.003)$, late revascularization $(P=0.020)$, and composite MACE $(P<0.001)$. Any degree of CAD in the LCX was associated with higher rates of MI than no LCX disease $(P=0.001)$.
Nonobstructive CAD in the RCA (Table 6) was associated with higher rates of chest pain reevaluation compared with obstructive RCA CAD and no RCA CAD (15\% versus $12.3 \%$ versus $9.8 \%$, resp. $P=0.016)$. Nonobstructive RCA CAD was also associated with higher rates of repeat testing $(P<0.001)$, average tests performed per patient $(P=0.001)$, and per patient testing cost $(P<0.001)$. Obstructive RCA CAD was associated with higher rates of hospitalization at time of reevaluation $(P=0.014)$, hospital LOS $(P=0.018)$, stroke $(P<0.001)$, MI $(P=0.001)$, late revascularization $(P<0.001)$, and composite MACE $(P<0.001)$.

One hundred seventy-four patients with obstructive CAD on CCTA were further broken down into 1-vessel disease, 
TABLE 5: Baseline demographic data, outcomes, and cost analysis based on burden of CAD in the LCX.

\begin{tabular}{|c|c|c|c|c|}
\hline & $\begin{array}{c}\text { No LCX CAD } \\
n=1029\end{array}$ & $\begin{array}{c}\text { Nonobstructive LCX } \\
n=441\end{array}$ & $\begin{array}{c}\text { Obstructive LCX } \\
n=48\end{array}$ & $P$ value \\
\hline Age & $51.3 \pm 14.2$ & $56.5 \pm 13.2$ & $62.9 \pm 13.6$ & $<0.001$ \\
\hline Male gender & $610(59.3)$ & $270(61.2)$ & $39(81.3)$ & 0.009 \\
\hline Chest pain reevaluation, $n(\%)$ & $104(10.1)$ & $62(14.1)$ & $8(16.7)$ & 0.048 \\
\hline Time to reevaluation, days & $358 \pm 345$ & $322 \pm 311$ & $441 \pm 400$ & 0.586 \\
\hline Hospitalization at reevaluation, $n(\%)$ & $47(4.6)$ & $34(7.7)$ & $4(8.3)$ & 0.039 \\
\hline Hospital LOS, days & $1.2 \pm 0.7$ & $1.4 \pm 0.9$ & $1.5 \pm 0.6$ & 0.382 \\
\hline Repeat testing, $n(\%)$ & $49(4.8)$ & $34(7.7)$ & $7(14.6)$ & 0.003 \\
\hline Tests per patient, mean & $0.05 \pm 0.3$ & $0.1 \pm 0.4$ & $0.2 \pm 0.5$ & 0.001 \\
\hline Cost per patient, mean & $\$ 15 \pm 85$ & $\$ 35 \pm 144$ & $\$ 75 \pm 201$ & $<0.001$ \\
\hline All-cause mortality, $n(\%)$ & $3(0.3)$ & $0(0)$ & $1(2.1)$ & 0.027 \\
\hline Stroke, $n(\%)$ & $4(0.4)$ & $7(1.6)$ & $2(4.2)$ & 0.003 \\
\hline MI, $n(\%)$ & $4(0.4)$ & $11(2.5)$ & $1(2.1)$ & 0.001 \\
\hline Late revascularization, $n(\%)$ & $1(0.1)$ & $3(0.7)$ & $1(2.1)$ & 0.020 \\
\hline Composite MACE, $n(\%)$ & $12(1.2)$ & $17(3.9)$ & $5(10.4)$ & $<0.001$ \\
\hline
\end{tabular}

LCX: left circumflex coronary artery.

TABLE 6: Baseline demographic data, outcomes, and cost analysis based on burden of CAD in the RCA.

\begin{tabular}{|c|c|c|c|c|}
\hline & $\begin{array}{c}\text { No RCA CAD } \\
n=990\end{array}$ & $\begin{array}{c}\text { Nonobstructive RCA } \\
n=454\end{array}$ & $\begin{array}{c}\text { Obstructive RCA } \\
n=73\end{array}$ & $P$ value \\
\hline Age & $51.2 \pm 14.1$ & $56.6 \pm 13.3$ & $59.6 \pm 14.0$ & $<0.001$ \\
\hline Male gender & $599(60.5)$ & $268(59)$ & $51(69.9)$ & 0.213 \\
\hline Chest pain reevaluation, $n(\%)$ & $97(9.8)$ & $68(15)$ & $9(12.3)$ & 0.016 \\
\hline Time to reevaluation, days & $371 \pm 349$ & $326 \pm 327$ & $277 \pm 215$ & 0.567 \\
\hline Hospitalization at reevaluation, $n(\%)$ & $43(4.3)$ & $36(7.9)$ & $6(8.2)$ & 0.014 \\
\hline Hospital LOS, days & $1.2 \pm 0.7$ & $1.3 \pm 0.6$ & $2.2 \pm 1.5$ & 0.018 \\
\hline Repeat testing, $n(\%)$ & $41(4.1)$ & $43(9.5)$ & $6(8.2)$ & $<0.001$ \\
\hline Tests per patient, mean & $0.05 \pm 0.3$ & $0.11 \pm 0.4$ & $0.08 \pm 0.3$ & 0.001 \\
\hline Cost per patient, mean & $\$ 14 \pm 88$ & $\$ 40 \pm 146$ & $\$ 34 \pm 125$ & $<0.001$ \\
\hline All-cause mortality, $n(\%)$ & $3(0.3)$ & $0(0)$ & $1(1.4)$ & 0.098 \\
\hline Stroke, $n(\%)$ & $4(0.4)$ & $4(0.9)$ & $5(6.8)$ & $<0.001$ \\
\hline MI, $n(\%)$ & $4(0.4)$ & $9(2.0)$ & $3(4.1)$ & 0.001 \\
\hline Late revascularization, $n(\%)$ & $0(0)$ & $3(0.7)$ & $2(2.7)$ & $<0.001$ \\
\hline Composite MACE, $n(\%)$ & $0(0)$ & $1(0.2)$ & $1(1.4)$ & $<0.001$ \\
\hline
\end{tabular}

RCA: right coronary artery.

2-vessel disease, or 3-vessel disease. Obstructive LM disease was grouped under 2-vessel disease for the purposes of this analysis. Table 7 outlines the demographics and outcomes in this subgroup. There was an age difference observed between the groups with older patients tending to have more diffuse disease $(P<0.001)$. Male predominance was observed in patients with 3-vessel disease when compared to the other two groups $(P=0.041)$. Reevaluations for chest pain were found more commonly in patients with 2-vessel CAD when compared to 1 -vessel and 3 -vessel $(22.7 \%$ versus $17.7 \%$ and $4.8 \%, P=0.009$ ). This increased incidence of reevaluation did not translate into a difference with regard to frequency of hospitalization, hospital LOS, frequency of repeat ischemic testing, or total number of repeat tests ordered per group.
Additionally, the time to reevaluation and the incidence of morbidity and mortality were not different between the three groups. Within the obstructive disease cohort, twenty-one patients underwent repeat testing at the time of reevaluation when compared with 10 patients where testing was deferred (67.7\% versus $32.3 \%$ ). No difference was observed with regard to stroke (9.5\% versus $0 \%, P=0.313)$, MI (9.5\% versus $10 \%$, $P=0.967)$, late revascularization ( $19 \%$ versus $0 \%, P=0.277$ ), and composite MACE $(33.3 \%$ versus $10 \%, P=0.222)$ whether additional testing was performed or not (Table 8). There were no mortalities observed in the obstructive CAD cohort.

Testing frequency also appeared to be affected by CCTA disease burden. When comparing all patients with reevaluation for chest pain, the average number of total tests 
TABLE 7: Outcomes in patients with obstructive CAD.

\begin{tabular}{|c|c|c|c|c|}
\hline & $\begin{array}{c}1 \mathrm{v} \text { CAD } \\
n=113\end{array}$ & $\begin{array}{c}2 \mathrm{v} \text { CAD } \\
n=44\end{array}$ & $\begin{array}{c}3 \mathrm{v} \text { CAD } \\
n=21\end{array}$ & $P$ value \\
\hline Age, years & $56.7 \pm 9.7$ & $59.5 \pm 14.3$ & $66.1 \pm 15.1$ & $<0.001$ \\
\hline Male, $n(\%)$ & $76(67.3)$ & $27(61.4)$ & $18(85.7)$ & 0.041 \\
\hline Chest pain reevaluation, $n(\%)$ & $20(17.7)$ & $10(22.7)$ & $1(4.8)$ & 0.009 \\
\hline Time to reevaluation, days & $330 \pm 326$ & $345 \pm 375$ & $181 \pm 0$ & 0.955 \\
\hline Hospitalization at reevaluation, $n(\%)$ & $11(55)$ & $6(60)$ & $1(100)$ & 0.665 \\
\hline Hospital LOS, days & $1.4 \pm 0.7$ & $1.8 \pm 0.7$ & $2.0 \pm 0.0$ & 0.272 \\
\hline Repeat testing, $n(\%)$ & $12(60)$ & $8(80)$ & $1(100)$ & 0.146 \\
\hline Total number of repeat tests, $n$ & 20 & 10 & 1 & 0.122 \\
\hline All-cause mortality, $n(\%)$ & $0(0)$ & $0(0)$ & $0(0)$ & $\mathrm{n} / \mathrm{a}$ \\
\hline Stroke, $n(\%)$ & $2(10)$ & $0(0)$ & $0(0)$ & 0.555 \\
\hline MI, $n(\%)$ & $2(10)$ & $1(10)$ & $0(0)$ & 0.946 \\
\hline Composite MACE, $n(\%)$ & $4(20)$ & $1(10)$ & $0(0)$ & 0.283 \\
\hline
\end{tabular}

lv: single-vessel Coronary artery disease (CAD); 2v: 2-vessel CAD; 3v: 3-vessel CAD; LOS: length of stay; MI: myocardial infarction; composite MACE: combined endpoint to all-cause mortality, stroke, MI, and late revascularization.

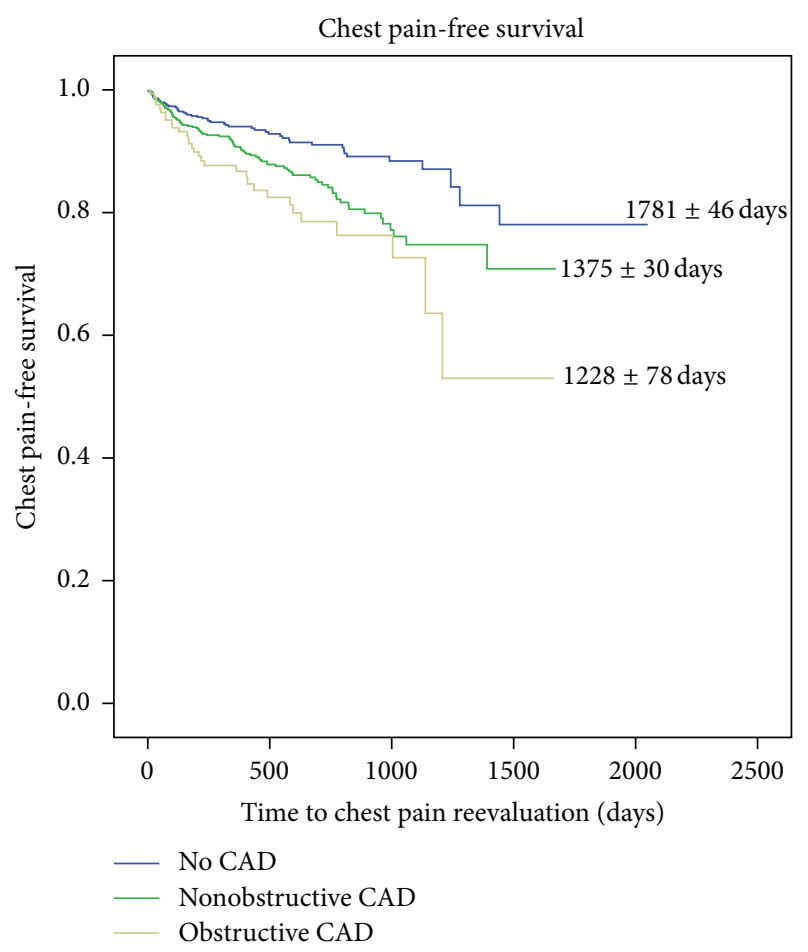

Figure 1: Kaplan-Meier curve demonstrating survival free from chest pain reevaluation based on CCTA disease burden.

ordered was higher in the patients with obstructive disease $(0.8 \pm 0.7)$ when compared to patient with nonobstructive CAD or no CAD $(0.7 \pm 0.7$ versus $0.4 \pm 0.5, P=0.006)$. Table 2 outlines approximate cost per test ordered. This resulted in a higher per patient cost in the obstructive CAD population when compared to nonobstructive CAD and no CAD patients ( $\$ 255$ versus $\$ 226$ versus $\$ 98, P=0.001$ ). However, once patients were selected to undergo additional testing, there was no difference amongst the groups with regard to the average number of tests ordered per patient in

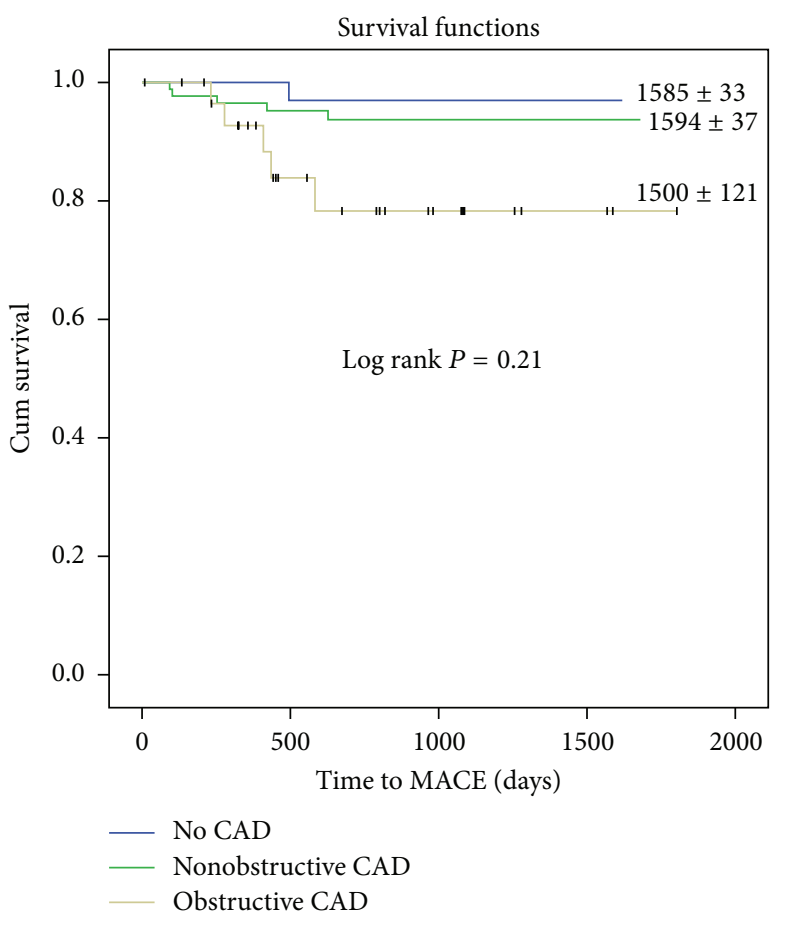

Figure 2: Kaplan-Meier curve demonstrating MACE free survival within the chest pain reevaluation population based on CCTA disease burden.

the obstructive, nonobstructive, and no CAD groups (1.2 \pm 0.5 versus $1.2 \pm 0.4$ versus $1.1 \pm 0.2, P=0.380$ ). Hospital LOS was not different between the groups and thus was not included in the cost analysis. Outcomes were not different in the 90 patients that underwent repeat testing at time of reevaluation for chest pain when compared to the 84 patients where testing was deferred (Table 9). No deaths were observed and the rates stroke $(2.2 \%$ versus $1.2 \%, P=1.0)$, MI (2.2\% versus $3.6, P=0.673)$, late revascularization $(4.4 \%$ versus $0 \%, P=0.122)$, and composite MACE $(7.8 \%$ versus 
TABLE 8: Outcomes in all patients undergoing reevaluation for recurrent chest pain based on whether repeat testing was performed at any point during the follow-up period.

\begin{tabular}{lccc}
\hline & $\begin{array}{c}\text { Repeat testing } \\
n=90(\%)\end{array}$ & $\begin{array}{c}\text { No repeat } \\
\text { testing } \\
n=84(\%)\end{array}$ & $P$ value \\
\hline All-cause mortality & $0(0)$ & $0(0)$ & $\mathrm{n} / \mathrm{a}$ \\
Stroke & $2(2.2)$ & $1(1.2)$ & 1.0 \\
MI & $2(2.2)$ & $3(3.6)$ & 0.673 \\
Late revascularization & $4(4.4)$ & $0(0)$ & 0.122 \\
Composite MACE & $7(7.8)$ & $4(4.8)$ & 0.538 \\
\hline
\end{tabular}

MI: myocardial infarction; composite MACE: combined endpoint to allcause mortality, stroke, MI, and late revascularization.

TABLE 9: Obstructive CAD patient outcomes (1v, 2v, or 3v CAD) based on whether patients were retested at any point during the follow-up period.

\begin{tabular}{lccc}
\hline & $\begin{array}{c}\text { Repeat testing } \\
n=21(\%)\end{array}$ & $\begin{array}{c}\text { No repeat } \\
\text { testing } \\
n=10(\%)\end{array}$ & $P$ value \\
\hline All-cause mortality & $0(0)$ & $0(0)$ & $\mathrm{n} / \mathrm{a}$ \\
Stroke & $2(9.5)$ & $0(0)$ & 0.313 \\
MI & $2(9.5)$ & $1(10)$ & 0.967 \\
Late revascularization & $4(19)$ & $0(0)$ & 0.277 \\
Composite MACE & $7(33.3)$ & $1(10)$ & 0.222 \\
\hline
\end{tabular}

MI: myocardial infarction; composite MACE: combined endpoint to allcause mortality, stroke, MI, and late revascularization.

$4.8 \%, P=0.538)$ were not different in the repeat testing and no repeat testing groups, respectively.

\section{Discussion}

This retrospective analysis illustrates that a previous CCTA demonstrating the absence of CAD or nonobstructive disease is associated with a reduction in both emergency department and outpatient reevaluations for chest pain in addition to MACE. In addition to improved outcomes, we demonstrated a reduction in additional testing and per patient cost based on overall CCTA disease burden and individual epicardial coronary vessel CAD burden and stratified by 1-vessel, 2vessel, and 3-vessel obstructive CAD. These findings further our understanding of both the prognostic utility and cost savings associated with the use of CCTA in acute and subacute chest pain evaluations.

Coronary artery disease is a major contributor of morbidity and mortality and effective means of evaluating those at increased risk of major cardiac events is paramount. At the same time, chest pain is a very common cause of emergency department (ED) presentations and return visits are common; therefore, an effective strategy to curtail these recurrent presentations is vital. CCTA has proven to be an effective means to evaluate patients with possible ACS in the emergency department who have low to intermediate risk of CAD [14]. Current 64-slice multidetector CTs have been shown to have a negative predictive value of approximately 99\% [8]. From a prognostic standpoint, previous studies have shown that those who have the absence of CAD or nonobstructive disease on CCTA (defined as lesions causing less than $50 \%$ luminal stenosis) in the evaluation of acute chest pain have equally benign clinical outcomes during follow-up evaluations [11]. In opposition, the CONFIRM registry showed that those individuals with obstructive 2- or 3-vessel disease or obstructive left main disease had higher rates of all-cause mortality and nonfatal MI despite absence of symptoms [1].

Given the high costs associated with recurrent ED evaluations and inpatient admissions for chest pain, a more definitive strategy of excluding significant CAD was needed and CCTA seems to be providing this necessity. A recently published study comparing CCTA to standard evaluation in the emergency department triage of low-risk chest pain revealed that CCTA led to a statistically significant decrease in admission rates, and return visits to the ED for recurrent chest discomfort were 5 times more likely within 30 days in the standard evaluation arm [12]. The standard evaluation arm consisted of patients either discharged directly from $\mathrm{ED}$ after evaluation or inpatient admission \pm stress testing.

Further studies have been done comparing CCTA to myocardial perfusion imaging in the evaluation of acute, low-risk chest pain patients in the ED. A recent multicenter randomized trial showed that compared to MPI, CCTA resulted in a statistically significant reduction in time to diagnosis and decreased health care costs without a difference in major adverse cardiac events after normal index findings [6].

CCTA not only allows prognostication based on presence or absence of obstructive disease but also allows for determination of plaque characterization that may reveal future presentations for chest pain due to acute coronary syndromes (ACS) [15]. These characteristics include "spotty" calcification, positive remodeling (defined as overall vessel diameter greater than $10 \%$ above reference vessel), and lower plaque attenuation less than 30 Hounsfield units [16].

Individual epicardial vessel CAD burden in this study demonstrated predictive utility for predicting repeat chest pain evaluations with the exception of LM obstructive disease. While this finding is unanticipated, it likely represents the very small number of patients ( 7 total patients) in the study cohort with obstructive LM CAD. Additionally, nearly all of these patients went on to coronary artery bypass graft (CABG) surgery within 30 days of initial CCTA. Thus with complete revascularization, lower recurrence rates for angina would be expected. An unpublished analysis by our group demonstrated the presence of LM coronary artery calcium (CAC) may predict MACE events to include stroke above that which would be predicted by total CAC score alone in a symptomatic cohort of patients. Indeed this current analysis also suggests the presence of obstructive LM disease predicts higher MACE rates to include mortality, stroke, MI, and late revascularization. 


\section{Conclusion}

Absence of obstructive disease on CCTA is associated with much lower rates of subsequent evaluations for chest pain and repeat testing with associated low MACE event rates over 6year review period and median followup of 22 months.

\section{Ethical Approval}

All authors hereby declare that all experiments have been examined and approved by the appropriate ethics committee and have therefore been performed in accordance with the ethical standards laid down in the 1964 Declaration of Helsinki. This project and the published data have been cleared for publication by the Department of Clinical Investigation, Brooke Army Medical Center.

\section{Consent}

All authors declare that IRB waiver for written informed consent for the purpose of data collection and analysis was granted prior to being engaged in data evaluation. IRB approval was granted for publication of this paper and accompanying images.

\section{Conflict of Interests}

The authors have declared that no competing interests exist.

\section{Authors' Contribution}

All authors were involved in study design and data collection, performed the statistical analysis, wrote the protocol, and wrote the first draft of the paper. All authors read and approved the final paper.

\section{Acknowledgments}

This work was performed as part of an approved IRB project in the Department of Cardiology at Brooke Army Medical Center under the review of the United States Army and United States Air Force. The opinions in this paper do not constitute endorsement by San Antonio Army Medical Center, the U.S. Army Medical Department, the U.S. Army Office of the Surgeon General, the Department of the Army, Department of Defense, or the U.S. Government of the information contained therein.

\section{References}

[1] I. Cho, H. J. Chang, J. M. Sung, M. J. Pencina, F. Y. Lin, A. M. Dunning et al., "Coronary computed tomographic angiography and risk of all-cause mortality and nonfatal myocardial infarction in subjects without chest pain syndrome form the CONFIRM registry (Coronary CT angiography evaluation for clinical outcomes: an international multicenter registry)," Circulation, vol. 126, pp. 304-313, 2012.
[2] J. A. Goldstein, M. J. Gallagher, W. W. O’Neill, M. A. Ross, B. J. O'Neil, and G. L. Raff, "A randomized controlled trial of multislice coronary computed tomography for evaluation of acute chest pain," Journal of the American College of Cardiology, vol. 49 , no. 8, pp. 863-871, 2007.

[3] H. I. Litt, C. Gatsonis, B. Snyder et al., "CT angiography for safe discharge of patients with possible acute coronary syndromes," The New England Journal of Medicine, vol. 366, no. 15, pp. $1393-$ 1403, 2012.

[4] U. Hoffmann, Q. A. Truong, D. A. Schoenfeld, E. T. Chou, P. K. Woodard, J. T. Nagurney et al., "Coronary CT angiography versus standard evaluation in acute chest pain," The New England Journal of Medicine, vol. 367, pp. 299-308, 2012.

[5] R. Blankstein, W. Ahmed, F. Bamberg, I. S. Rogers, C. L. Schlett, K. Nasir et al., "Comparison of exercise treadmill testing with cardiac computed tomography angiography among patients presenting to the emergency room with chest pain: the Rule Out Myocardial Infarction Using Computer-Assisted Tomography (ROMICAT) study," Circulation: Cardiovascular Imaging, vol. 5, no. 2, pp. 233-242, 2012.

[6] J. A. Goldstein, K. M. Chinnaiyan, A. Abidov et al., "The CT-STAT (coronary computed tomographic angiography for systematic triage of acute chest pain patients to treatment) trial," Journal of the American College of Cardiology, vol. 58, no. 14, pp. 1414-1422, 2011.

[7] N. Patel, R. S. Pal, F. Flores, and M. Budoff, "Utility of cardiac computed tomography angiography to exclude clinically significant obstructive coronary artery disease in patients after myocardial perfusion imaging," American Journal of Cardiology, vol. 109, no. 2, pp. 165-168, 2012.

[8] M. J. Budoff, D. Dowe, J. G. Jollis et al., "Diagnostic performance of 64-multidetector row coronary computed tomographic angiography for evaluation of coronary artery stenosis in individuals without known coronary artery disease: results from the prospective Multicenter ACCURACY (Assessment by Coronary Computed Tomographic Angiography of Individuals Undergoing Invasive Coronary Angiography) trial," Journal of the American College of Cardiology, vol. 52, no. 21, pp. 1724-1732, 2008.

[9] A. J. Taylor, M. Cerqueira, J. M. Hodgson et al., "ACCF/SCCT/ ACR/AHA/ASE/ASNC/NASCI/SCAI/SCMR 2010 appropriate use criteria for cardiac computed tomography," Journal of the American College of Cardiology, vol. 56, no. 22, pp. 1864-1894, 2010.

[10] Centers for Medicare and Medicaid Services (CMS), HHS, "Medicare Program; hospital inpatient prospective payment systems for acute care hospitals and the long-term care hospital prospective payment system changes and FY2011 rates; provider agreements and supplier approvals; and hospital conditions of participation for rehabilitation and respiratory care services; Medicaid program: accreditation for providers of inpatient psychiatric services. Final rule," Federal register, vol. 75, no. 157, pp. 50041-50681, 2010.

[11] R. Beigel, D. Oieru, O. Goitein et al., "Usefulness of routine use of multidetector coronary computed tomography in the "Fast Track" evaluation of patients with acute chest pain," American Journal of Cardiology, vol. 103, no. 11, pp. 1481-1486, 2009.

[12] M. Poon, M. Cortegiano, A. J. Abramowicz, M. Hines, A. J. Singer, M. C. Henry et al., "Associations between routine coronary computed tomographic angiography and reduced unnecessary hospital admissions, length of stay, recidivism rates, and invasive coronary angiography in the emergency 
department triage of chest pain," Journal of the American College of Cardiology, vol. 62, no. 6, pp. 543-552, 2013.

[13] S. Abbara, A. Arbab-Zadeh, T. Q. Callister et al., "SCCT guidelines for performance of coronary computed tomographic angiography: a report of the Society of Cardiovascular Computed Tomography Guidelines Committee," Journal of Cardiovascular Computed Tomography, vol. 3, no. 3, pp. 190-204, 2009.

[14] D. A. Bluemke, S. Achenbach, M. Budoff et al., "Noninvasive coronary artery imaging: magnetic resonance angiography and multidetector computed tomography angiography: a scientific statement from the American Heart Association committee on cardiovascular imaging and intervention of the council on cardiovascular radiology and intervention, and the councils on clinical cardiology and cardiovascular disease in the young," Circulation, vol. 118, no. 5, pp. 586-606, 2008.

[15] K. M. Woods, C. Fischer, M. K. Cheezum, E. A. Hulten, B. Nguyen, and T. C. Villines, "The prognostic significance of coronary CT angiography," Current Cardiology Reports, vol. 14, no. 1, pp. 7-16, 2012.

[16] S. Motoyama, M. Sarai, H. Harigaya et al., "Computed tomographic angiography characteristics of atherosclerotic plaques subsequently resulting in acute coronary syndrome," Journal of the American College of Cardiology, vol. 54, no. 1, pp. 49-57, 2009. 


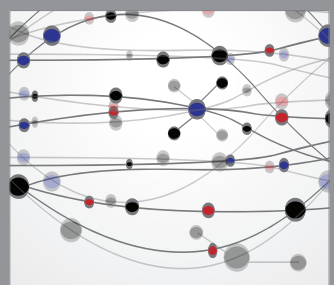

The Scientific World Journal
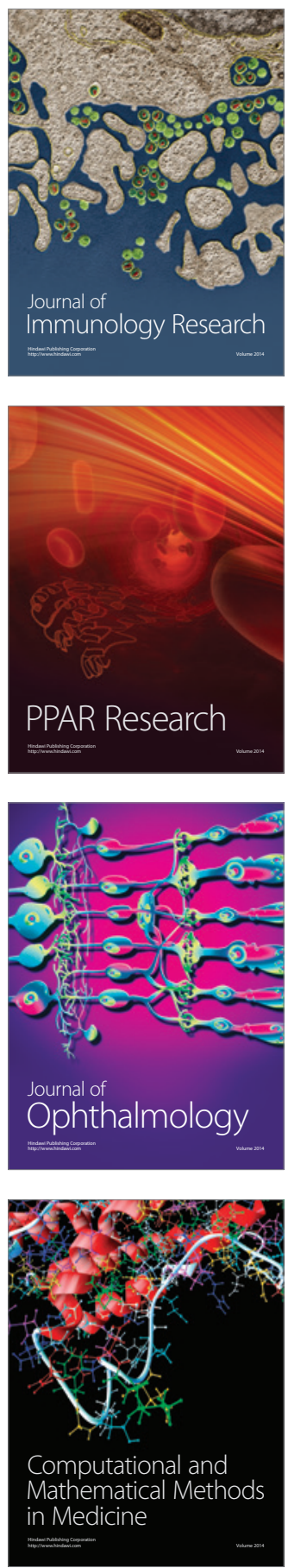

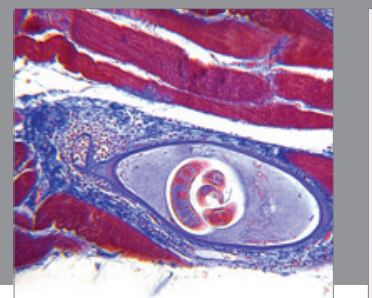

Gastroenterology

Research and Practice
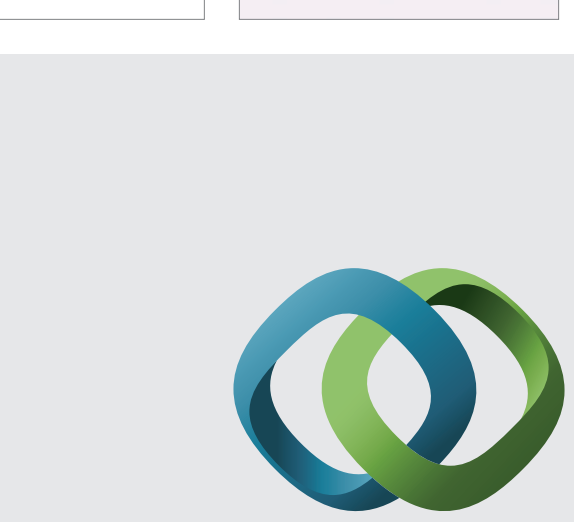

\section{Hindawi}

Submit your manuscripts at

http://www.hindawi.com
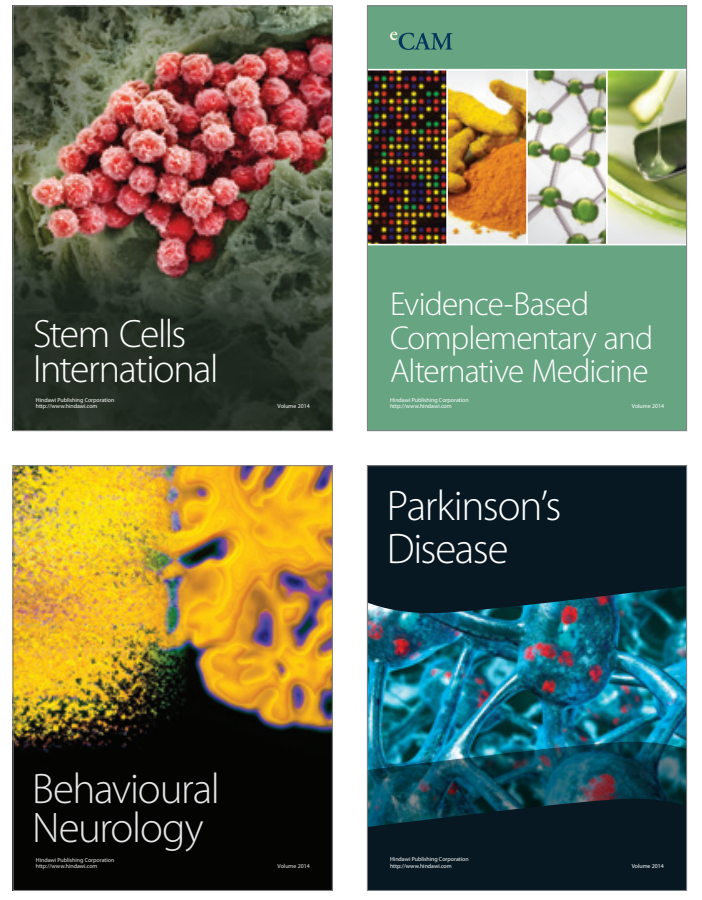
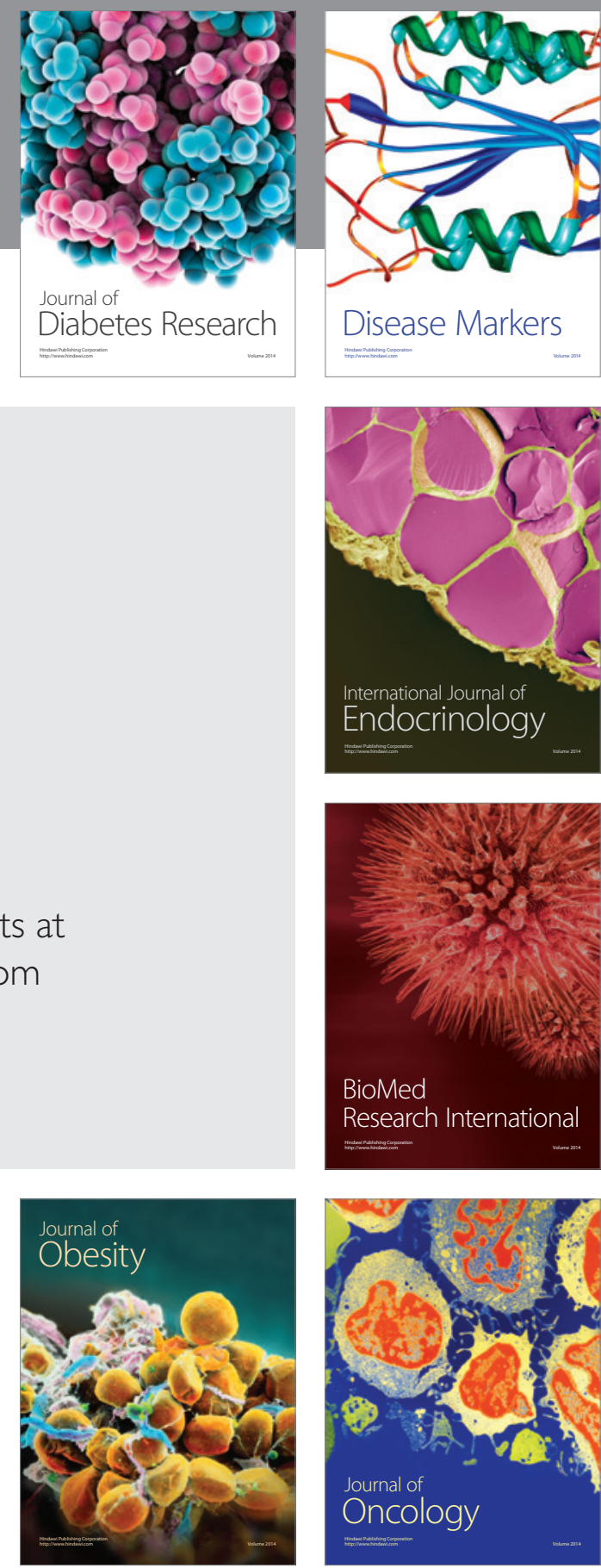

Disease Markers
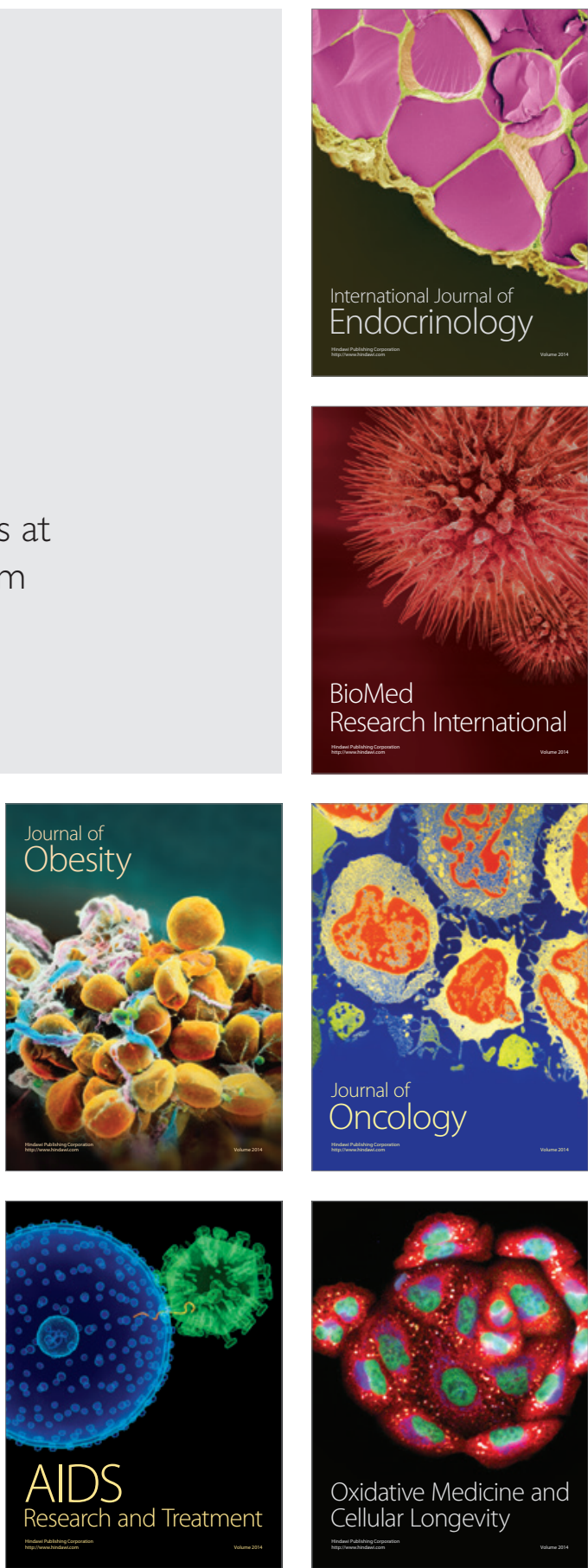